\title{
Genome-scale analyses of transcriptional start sites in Mycobacterium marinum under normoxic and hypoxic conditions
}

\author{
Shaojia Huang ${ }^{1,2}$, Wei Zhou ${ }^{1,2}$, Wei Tang ${ }^{1}$, Yong Zhang ${ }^{1}$, Yangbo Hu${ }^{1 *}$ and Shiyun Chen ${ }^{1 *}$
}

\begin{abstract}
Background: Hypoxic stress plays a critical role in the persistence of Mycobacterium tuberculosis (Mtb) infection, but the mechanisms underlying this adaptive response remain ill defined.

Material and methods: In this study, using M. marinum as a surrogate, we analyzed hypoxic responses at the transcriptional level by Cappable-seq and regular RNA-seq analyses.

Results: A total of 6808 transcriptional start sites (TSSs) were identified under normoxic and hypoxic conditions. Among these TSSs, 1112 were upregulated and 1265 were downregulated in response to hypoxic stress. Using SigE-recognized consensus sequence, we identified 59 SigE-dependent promoters and all were upregulated under hypoxic stress, suggesting an important role for SigE in this process. We also compared the performance of Cappable-seq and regular RNA-seq using the same RNA samples collected from normoxic and hypoxic conditions, and confirmed that Cappable-seq is a valuable approach for global transcriptional regulation analyses.

Conclusions: Our results provide insights and information for further characterization of responses to hypoxia in mycobacteria, and prove that Cappable-seq is a valuable approach for global transcriptional studies in mycobacteria.
\end{abstract}

\section{Introduction}

Mycobacterium tuberculosis (Mtb), the etiologic agent of tuberculosis (TB), is one of the most successful bacterial pathogens of humans. It is estimated that one-third of the world's population is infected by Mtb, of whom $90 \%$ will develop latent TB infection (LTBI) [1], presenting a heavy burden for TB prevention. Through inhalation of aerosol droplets containing bacilli, Mtb reaches lung airways and is engulfed by alveolar macrophages, which then recruit mononuclear cells and $\mathrm{T}$ lymphocytes to form granulomas [2]. In both macrophages and

\footnotetext{
* Correspondence: ybhu@wh.iov.cn; sychen@wh.iov.cn

'CAS Key Laboratory of Special Pathogens and Biosafety, Wuhan Institute of Virology, Center for Biosafety Mega-Science, Chinese Academy of Sciences, Wuhan 430071, China

Full list of author information is available at the end of the article
}

granulomas, Mtb encounters hypoxic stress [3, 4], which has been shown to be important for inducing the transition to dormancy, a nonreplicating and drug-resistant state $[1,5]$.

Several in vitro models have been established to investigate how Mtb adapts to hypoxic stress, such as the Wayne model [6] and the defined hypoxia model [7, 8]. In the Wayne model, bacterial cells are grown in sealed and stirred tubes with a defined headspace-to-culture ratio, allowing the gradual exhaustion of oxygen [6]. Compared with the defined hypoxia model, which requires a constant flow of low oxygen gas over the culture to maintain hypoxia $[7,8]$, the Wayne model is relatively simple to set up in the laboratory $[5,6,9]$.

It has been reported that the two-component response regulator DosR is critical for the initial hypoxic response $[8,10]$, and hundreds of genes are

(c) The Author(s). 2021 Open Access This article is licensed under a Creative Commons Attribution 4.0 International License, which permits use, sharing, adaptation, distribution and reproduction in any medium or format, as long as you give appropriate credit to the original author(s) and the source, provide a link to the Creative Commons licence, and indicate if changes were made. The images or other third party material in this article are included in the article's Creative Commons licence, unless indicated otherwise in a credit line to the material. If material is not included in the article's Creative Commons licence and your intended use is not permitted by statutory regulation or exceeds the permitted use, you will need to obtain permission directly from the copyright holder. To view a copy of this licence, visit http://creativecommons.org/licenses/by/4.0/. The Creative Commons Public Domain Dedication waiver (http://creativecommons.org/publicdomain/zero/1.0/) applies to the data made available in this article, unless otherwise stated in a credit line to the data. 
subsequently upregulated independent of DosR, defining the enduring hypoxic response (EHR) [9]. The regulatory networks of Mtb during hypoxia and reaeration have been reconstructed based on ChIP-Seq analyses [11]. These studies suggest that transcriptional regulators play essential roles in hypoxic responses. In addition to transcriptional regulators, sigma factors have also been known to regulate gene transcription in stress responses. Sigma factor $(\sigma)$ first reconstitutes with the RNA polymerase (RNAP) core enzyme to form a holoenzyme, which can recognize specific promoter sequences in the transcription initiation process [12]. Bacterial promoters of the $\sigma^{70}$ family contain -10 and -35 elements located upstream of transcriptional start sites (TSSs) [13]. Therefore, global identification of TSSs would provide valuable information for promoter characterization. The Mtb genome encodes 13 sigma factors, most of which are critical for survival under stress conditions [14-18]. Among them, $\operatorname{sig} B$ and $\operatorname{sig} E$ have been shown to be upregulated under hypoxic stress [19], although their functions in hypoxic responses have not been completely characterized.

In recent studies, different methods have been developed for the global analysis of TSSs in bacteria, such as differential RNA-seq (dRNA-seq) [20] and Cappable-seq [21]. In prokaryotes, primary transcripts reflecting TSSs constitute less than $5 \%$ of the total RNA; the vast majority are processed ribosomal RNAs. Taking advantage of the distinctive $5^{\prime}$ triphosphate ends of primary transcripts, dRNA-seq and Cappable-seq use different strategies to enrich for primary transcripts. dRNA-seq relies on $5^{\prime}$ monophosphate-dependent terminator exonuclease (TEX) to deplete processed transcripts, thus enhancing the relative content of primary transcripts [20]. In Cappable-seq, vaccinia capping enzyme (VCE) is used to label $5^{\prime}$ triphosphorylated RNA with a biotin derivative, and then the primary transcripts are captured on streptavidin beads [21]. Global characterizations of TSSs in different mycobacterial species have also been documented [22-26], but the influences of different stresses on the strength of these TSSs in mycobacteria are not well defined.

M. marinum has been used as a surrogate for Mtb to overcome its slow-growing nature and to avoid the requirement of a biosafety level 3 (BSL-3) facility. More importantly, M. marinum also forms granulomas during zebrafish infection [27, 28]. In this study, we characterized the transcriptional regulation of $M$. marinum under normoxic and hypoxic conditions by quantifying the strength of TSSs using Cappable-seq [21], which provides insights and information for further characterization of hypoxic responses in mycobacteria.

\section{Results}

\section{Hypoxic treatment of $M$. marinum}

To test the hypoxic response of $M$. marinum, we applied the Wayne low-oxygen model to mimic hypoxic conditions [3, 6]. In this model, an oxygen-responsive dye, methylene blue, was added to the medium to indicate the depletion of oxygen. As shown in Fig. 1a, the dye changed from blue to colorless when incubated at $32^{\circ} \mathrm{C}$ for $24 \mathrm{~h}$. M. marinum cell cultures with and without hypoxic treatment were collected. Colony forming units (CFU) were increased approximately 3 -fold after $24 \mathrm{~h}$ under oxygen limitation (Fig. 1b), suggesting that the cells successfully coped with hypoxic stress. As positive controls, the induction of the dormancy regulator $\operatorname{dos} R$ and one of its activated genes, tgs1 [10], under hypoxic conditions was confirmed using qRT-PCR analysis. Consistent with a previous report [11], the expression level of $i n h A$, which is known to be involved in mycolic acid biosynthesis [29], was decreased (Fig. 1c).

To further confirm the hypoxic responses of $M$. marinum in the Wayne low oxygen model, we performed regular RNA-seq and found a total of 5112 and 5362 coding sequences (CDSs) with fragments per kilobase per million mapped fragments (FPKM) values of ten or more under normoxic and hypoxic conditions, respectively (Table S1). We observed numerous differentially expressed genes, among which 1087 were upregulated and 1791 were downregulated in response to hypoxic stress (Fig. 1d, Table S1). Several transcriptional regulators, such as $\operatorname{dos} R, \operatorname{sig} E$, and $\operatorname{sig} B$, were upregulated, whereas most ribosomal genes were downregulated (Fig. 1d). These results confirm that $M$. marinum cells experienced hypoxic stress in our Wayne model.

\section{Global mapping of TSSs in M. marinum under normoxic and hypoxic conditions}

To facilitate a detailed regulatory analysis of the transcriptional regulation of $M$. marinum under hypoxic stress, we next identified TSSs at the genome-scale under normoxic and hypoxic conditions by Cappableseq [21]. On average, over one million reads were uniquely mapped to the $M$. marinum genome outside of the ribosomal and tRNA regions. By analyzing the first nucleotide at the $5^{\prime}$ end of the mapped reads, we identified a total of 6808 TSSs (Fig. 1e, Table S2). Consistent with other reports [21, 22], purine bases were enriched at the +1 position $(A+G, 95.8 \%)$, and pyrimidine bases were enriched at the -1 position $(C+T, 72.8 \%)$ (Fig. 1f). Moreover, 2046 out of 6808 of TSSs identified in our study matched completely and 636 out of 6808 TSSs matched with $1 \mathrm{bp}$ or $2 \mathrm{bp}$ difference compared with data from a previous dRNA-seq analysis [24] (Table S2). These data corroborate that Cappable-seq is a valuable approach for TSS identification. 
A

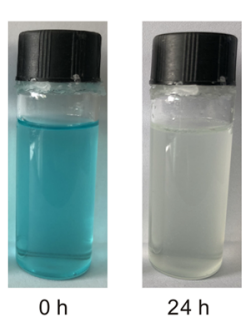

D

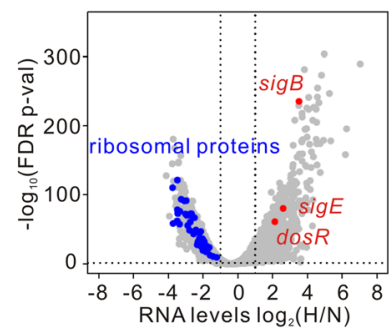

B

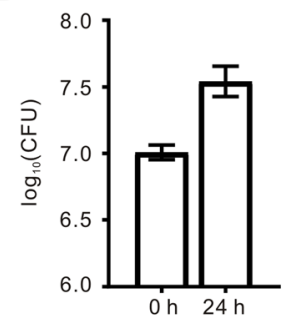

E
C

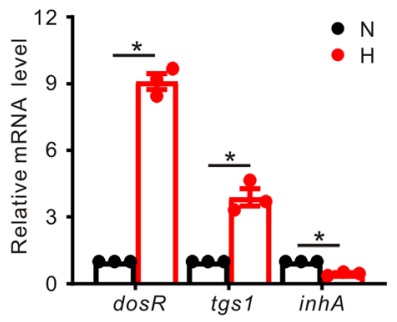

$\mathrm{F}$
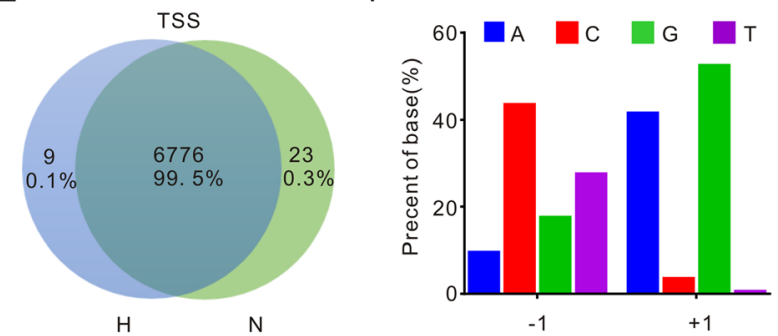

Fig. 1 Global mapping of TSSs under normoxic $(\mathrm{N})$ and hypoxic $(\mathrm{H})$ conditions. a M. marinum cultures prior to $(0 \mathrm{~h})$ and $24 \mathrm{~h}$ after hypoxic incubation. $\mathbf{b}$ The CFU numbers of M. marinum prior to and $24 \mathrm{~h}$ after hypoxic incubation. Data are shown as the mean \pm SEM values of three experiments performed in triplicate. c Transcriptional levels of $\operatorname{dos} R$, tgs 1 and inhA genes under normoxic and hypoxic conditions. Relative transcriptional levels of these genes under normoxic conditions were normalized to 1. Data are shown as the mean \pm SEM values of biological triplicates performed in duplicate. ${ }^{*}, p<0.05$. $\mathbf{d}$ Volcano plot showing the differential expression of global mRNA as a ratio of expression under hypoxic $(\mathrm{H})$ and normoxic $(\mathrm{N})$ conditions. The blue dots are downregulated genes encoding ribosomal proteins, and the red dots are upregulated dosR, sigE and sigB genes. Data are shown as the mean values of biological triplicates. FDR-adjusted $p$-values were obtained using DESeq2. e Venn diagram showing the numbers and percentages of TSSs identified under normoxia and hypoxia. $\mathbf{f}$ Percentages of base preference at the -1 and +1 (relative to TSSs) positions of 6808 TSSs identified under normoxia and hypoxia

\section{Characterization of $M$. marinum promoters}

To gain insight into the transcriptional organization of M. marinum, we classified TSSs into five categories according to genome position and TSS strength as previously reported [20]: primary TSS (pTSS), secondary TSS (sTSS), internal TSS (iTSS), antisense TSSs (aTSS) and orphan TSS (oTSS) (Fig. 2a and b). A total of 2959 pTSSs were identified, which covered more than half of the 5557 genes in M. marinum. Among them, 2490 pTSSs were found under both normoxic and hypoxic conditions, and 229 pTSS were identified only under hypoxic conditions (Fig. 2c), indicating stress-induced transcription. Moreover, a total of 1853 iTSSs, 1375 aTSSs, 385 oTSSs, and 1490 sTSSs were identified. Notably, some aTSSs and oTSSs may initiate the transcription of small noncoding RNAs or represent the products of spurious transcription initiation, which requires further investigation.

To characterize the common features of $M$. marinum promoters, we used $50 \mathrm{bp}$ sequences upstream of 2490 pTSSs to search for conserved motifs using MEME [30, 31]. A - 10 motif was found upstream of $80 \%$ (1988/ 2490) of the pTSSs, among which YANNNT (1547/ $2490,62 \%)$ was the most commonly identified sequence (Fig. 2d). We could not detect a consensus - 35 motif, which is consistent with previous reports in Mtb and $M$. smegmatis $[22,25,26]$. Furthermore, $\mathrm{T}_{-15} \mathrm{G}_{-14} \mathrm{~N}$ (TGN) has been described to form an extended - 10 consensus in several bacterial promoters, and $\mathrm{G}_{-14}$ or $\mathrm{G}_{-13}$ together with the -10 consensus has been characterized as a minimal promoter type in mycobacteria [32, 33]. We analyzed the frequency of TGN, $\mathrm{G}_{-14}$ or $\mathrm{G}_{-13}$ upstream of the -10 motif, and found that only $12 \%$ (193/ 1547) of YANNNT motifs were preceded by TGN and $88 \%(1368 / 1547)$ of YANNNT motifs were preceded by $\mathrm{G}_{-14}$ or $\mathrm{G}_{-13}$ (Fig. 2e). It is possible that the relatively high percentage of $G_{-14}$ or $G_{-13}$ motif compensates for the deficiency of the -35 motif in mycobacteria [33, 34].

\section{Widespread leaderless transcripts in M. marinum}

Although the mechanism of translation from leaderless mRNA has not been completely characterized, leaderless transcripts have been reported as a prominent feature in both $\mathrm{Mtb}$ and $M$. smegmatis [22, 25]. To determine whether this feature exists in $M$. marinum, we analyzed the length of the $5^{\prime}$ untranslated region ( $5^{\prime}$ UTR) associated with pTSSs. A large number of $5^{\prime}$ UTR sequences are shorter than $10 \mathrm{nt}$, suggesting a possible richness of leaderless RNAs in $M$. marinum (Fig. 3a). To characterize the relationship between $5^{\prime}$ UTR length and translational efficiency, we tested the expression of eGFP with 5' UTR lengths ranging from 0 to $12 \mathrm{nt}$ (Fig. 3b). 


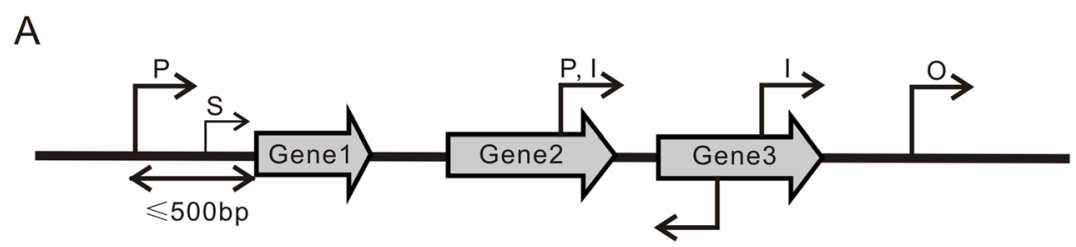

B

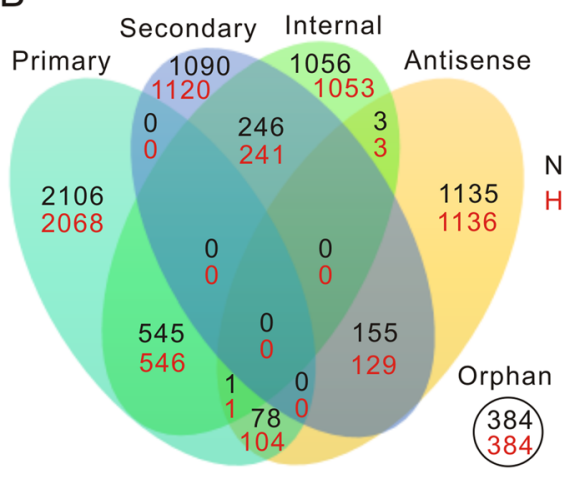

$\mathrm{D}$

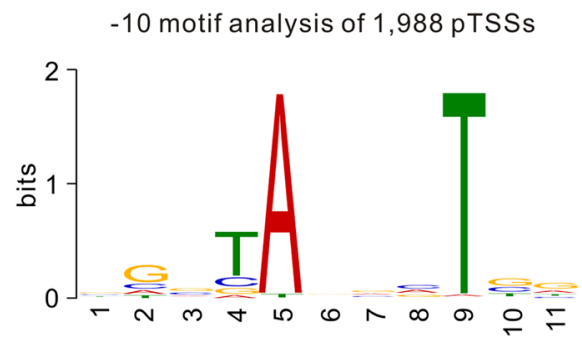

C

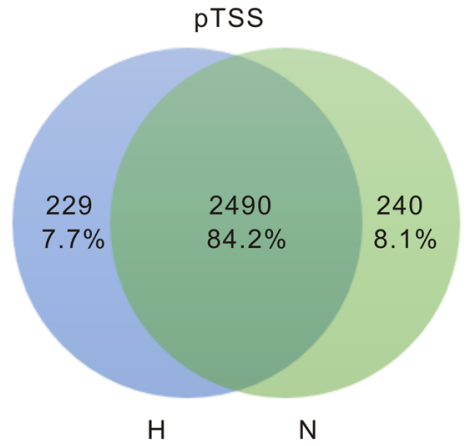

$\mathrm{E}$

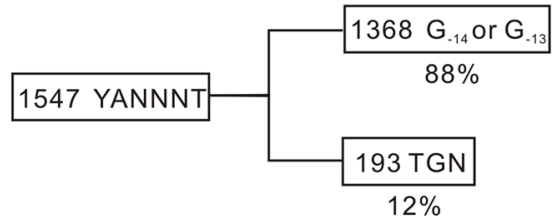

Fig. 2 Classifications of TSSs and the features of promoters in M. marinum. a Classification scheme for TSSs based on genome position and TSS strength: primary (P), secondary (S), internal (I), antisense (A) and orphan (O). b Venn diagrams showing classifications of TSSs identified under normoxic ( $\mathrm{N}$, black color) and hypoxic $(\mathrm{H}$, red color) conditions. A TSS could be classified into more than one category. $\mathbf{c}$ Venn diagram showing the numbers and percentages of pTSSs identified under normoxia and hypoxia. d Consensus for - 10 motif of 1988 promoters upstream of pTSSs expressed under both normoxia and hypoxia generated by MEME [30, 31]. Regions containing 12 bp ( -5 to -16 relative to TSSs) were used for analysis. e Numbers and percentages of $\mathrm{G}_{-14}$ or $\mathrm{G}_{-13}$ and TGN preceding the -10 YANNNT motif

Interestingly, we found that transcripts with a $5^{\prime}$ UTR less than $4 \mathrm{nt}$ could be efficiently translated (Fig. 3c), suggesting that transcripts with a $0-3$ nt 5 ' UTR could be defined as functional leaderless transcripts. Based on these analyses, we revealed that a total of 1288 TSSs initiate leaderless transcripts in M. marinum (Table S2), and 991 of these TSSs were reported [24]. Interestingly, $39.8 \%(1088 / 2730)$ and $38.4 \%$ (1045/2719) of pTSSs initiate functional leaderless transcripts under normoxia and hypoxia respectively (Fig. 3d).

\section{Transcriptional regulation in response to hypoxic stress}

By quantifying the strength of TSSs under normoxic and hypoxic conditions, we analyzed the global transcriptional regulation of $M$. marinum in hypoxic responses. Of the 6808 TSSs identified under either condition, 9 TSSs were specifically detected under hypoxic conditions and 23 TSSs were specifically detected under normoxic conditions (Fig. 1e, Table S3). In addition, 1103 TSSs were significantly upregulated and 1242 TSSs were significantly downregulated in response to hypoxic stress (fold change $\geq 2$, FDR $p$-value $<0.05$; Fig. 4a) (Table S2). Table 1 shows the top 15 upregulated and downregulated pTSS meeting the criterion of a relative read score (RRS) of 20 or more in all three replicates. Among them, the pTSS of $\operatorname{sig} B$ was in the top 15 upregulated pTSSs, which is consistent with our observation in regular RNA-seq data that the mRNA level of $\operatorname{sig} B$ was increased under hypoxic conditions. For the 15 most downregulated pTSSs, 2 genes downstream of these pTSSs encoded synthetases, which is consistent with the low metabolic activity observed under hypoxic conditions.

Altering the ratio of alternative sigma factor with primary sigma factor could regulate the transcription of many genes and is a common strategy used by bacteria to survive in diverse harsh conditions [35]. Consistent 


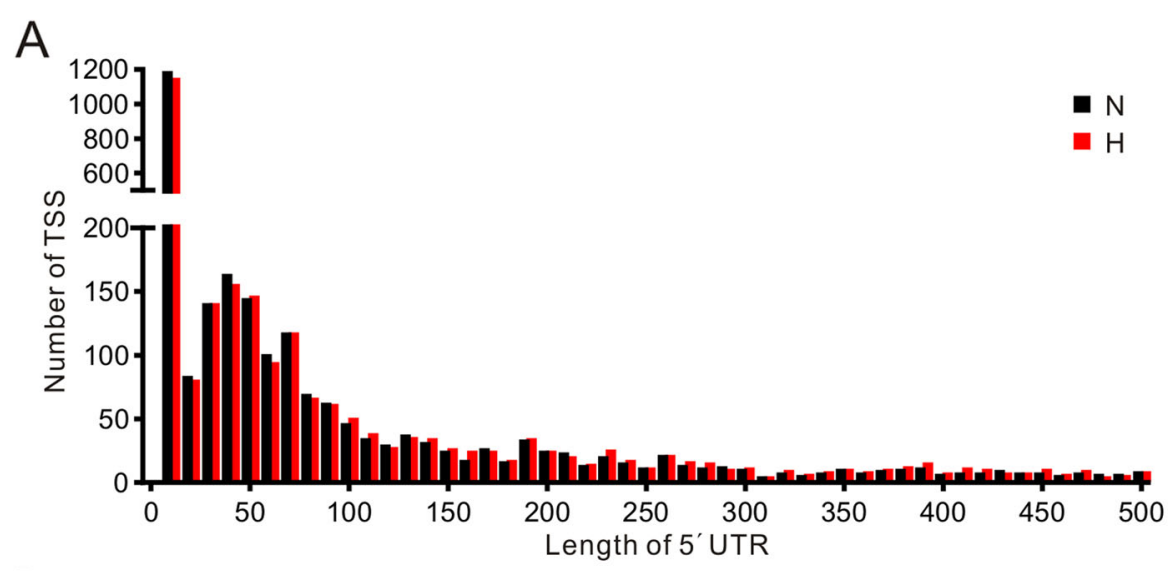

B

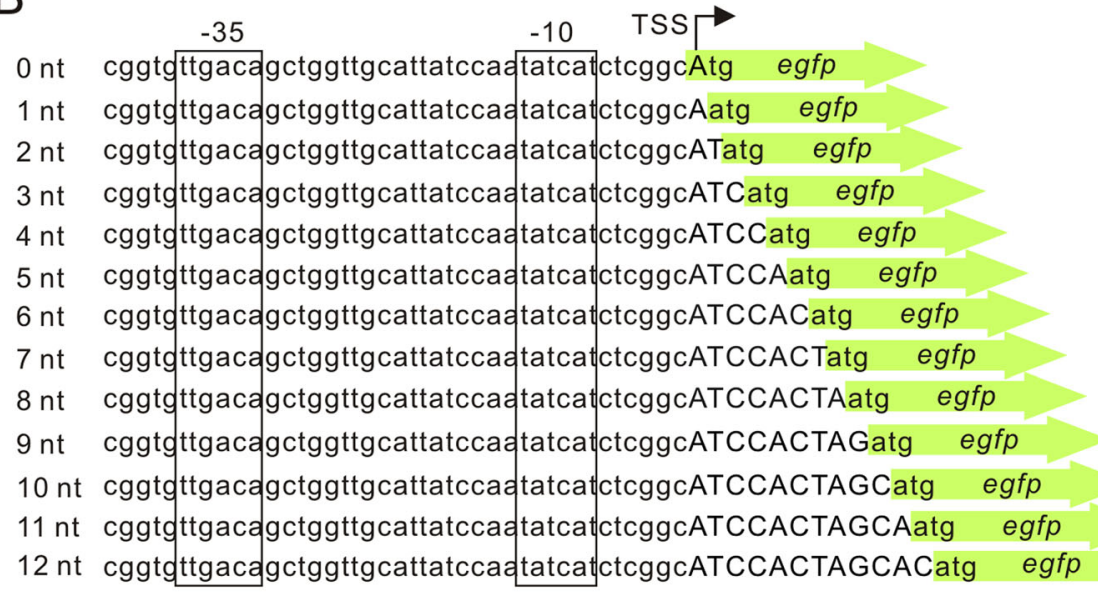

C
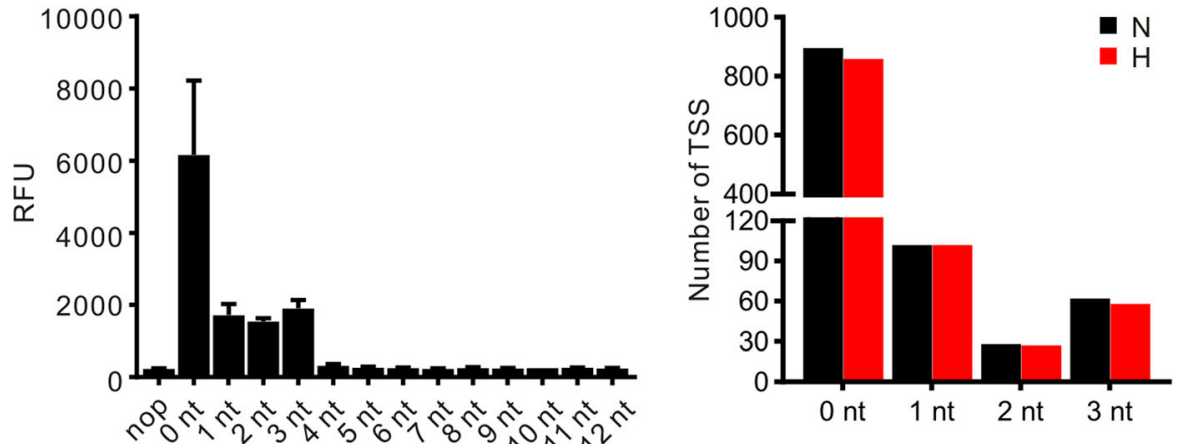

Fig. 3 Leaderless transcripts are common in M. marinum. a Distribution of $5^{\prime}$ UTR length (10 nt bins) of mRNAs with a primary TSS. Black and red bars indicate mRNAs associated with 2730 pTSSs and 2719 pTSSs identified under normoxia (N) and hypoxia (H), respectively. b Sequences of promoters and 5' UTRs of egfp transcribed with different lengths of 5' UTR. c Relative fluorescence units (RFU) of egfp transcribed with of 5' UTRs of different lengths, from $0 \mathrm{nt}$ to $12 \mathrm{nt}$. Data are shown as the mean \pm SD values from two independent colonies. "nop" indicates the background value for measuring RFU. $\mathbf{d}$ Numbers of pTSS initiating the transcription of functional leaderless transcripts under normoxic (N) and hypoxic (H) conditions

with previous reports that the expression of sigE and $\operatorname{sig} B$ was induced in Mtb under hypoxic stress [19], we observed that the pTSS of $\operatorname{sig} B$ was one of the most upregulated TSSs under hypoxic conditions in M. marinum (Table 1). As the promoter of the $\operatorname{sig} B$ gene was activated by SigE [36], we further analyzed whether other SigE-dependent promoters were activated under hypoxia. We took advantage of the reported SigEdependent promoter consensus GGGAACY-N $\mathrm{N}_{16-17^{-}}$ CGTT [37] to search for promoters recognized by SigE. 

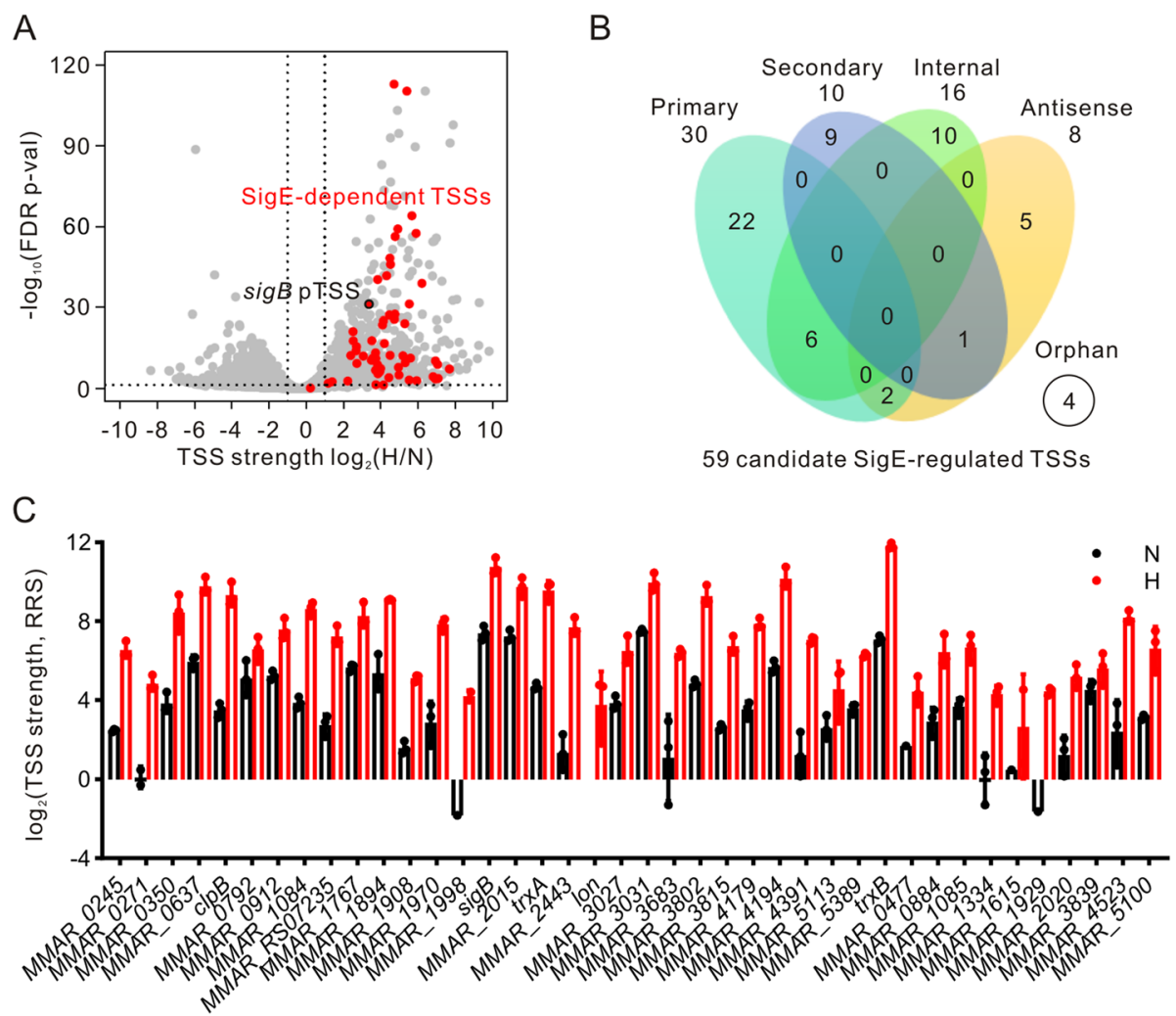

Fig. 4 Global characterization of transcriptional regulation in response to hypoxic stress. a Volcano plot showing fold-changes of TSS strength as a ratio of hypoxic $(\mathrm{H})$ and normoxic $(\mathrm{N})$ incubation. The red dots are 59 SigE-dependent TSSs, including the pTSS of sigB (marked). Data are shown as the mean values of biological triplicates. FDR-adjusted p-values were obtained using DESeq2. b Classification of 59 SigE-dependent TSSs. c TSS strength of SigE-dependent pTSSs and sTSSs under normoxia (N) and hypoxia (H). The first 30 genes are associated with pTSSs and the last 10 genes are associated with sTSSs. Data are shown as the mean \pm SD values of biological triplicates

In total, we obtained 59 TSSs of different categories (Fig. $4 \mathrm{~b})$ that were elevated in response to hypoxia (Table S2, Fig. 4a, c).

\section{Comparison of Cappable-seq and regular RNA-seq in global transcriptional regulation analyses}

We next compared the data in Cappable-seq with our data in regular RNA-seq to confirm the efficiency of Cappable-seq in the global analysis of transcriptional regulation. Among 2490 pTSSs expressed under both normoxia and hypoxia, 2387 corresponded to genes whose transcripts were detected in regular RNA-seq. We compared the fold-changes of RNA levels with TSS strength, and a moderate correlation was observed $(\mathrm{r}=$ 0.45; Fig. 5a). As expected, most of the genes showed similar patterns of regulation in both methods (Fig. 5a). The small differences observed may be due to posttranscriptional regulation or other unknown factors. Together, these analyses indicate that Cappable-seq is a valuable method for transcriptional analysis.

Inconsistencies between Cappable-seq and regular RNA-seq may represent genes that are subject to posttranscriptional regulation. Therefore, we selected genes with a ratio of fold-change $(\mathrm{H} / \mathrm{N})$ of RNA levels to fold-change $(\mathrm{H} / \mathrm{N})$ of TSS strength greater than 3 or less than 0.33 . Interestingly, a total of 568 genes (Fig. 5a), including 393 upregulated genes (ratio $>3$ ) and 175 downregulated genes (ratio $<0.33$ ), were suggested to be regulated at the posttranscriptional level under hypoxic conditions. Considering its function and homology to Mtb, we chose one of upregulated gene, fadA5, to validate posttranscriptional regulation. FadA 5 is involved in fatty acid metabolism and is a leaderless transcript in both $M$. marinum and Mtb [22]. We respectively constructed the fadA5 promoter and translational fusions to egfp using the integrative vector pMV306 [38]. M. marinum carrying egfp fusion plasmids was grown under normoxia and hypoxia. In qRT-PCR assays to detect egfp mRNA levels, we did not observe a significant difference in $f a d A 5$ promoter activity in response to hypoxic stress (Fig. 5b), but the level of the translational fusion fadA5:: egfp increased 1.5-fold in response to hypoxic stress (Fig. $5 \mathrm{c})$. These results suggested that the stability of fadA5 mRNA might be increased under hypoxic conditions 
Table 1 Top 15 pTSSs up- or downregulated under hypoxic stress

\begin{tabular}{|c|c|c|c|}
\hline Position & Associated gene & $\mathrm{FC}(\mathrm{H} / \mathrm{N})$ & Product \\
\hline \multicolumn{4}{|l|}{ upregulated } \\
\hline $2,741,615,+$ & $\operatorname{trx} \times 1$ & 30.3 & thioredoxin \\
\hline $5,166,688,+$ & kgd & 23.1 & multifunctional oxoglutarate decarboxylase \\
\hline $5,928,771,+$ & MMAR_4874 & 23.1 & transcriptional regulator \\
\hline $19,429,+^{a}$ & MMAR_p23 & 23.0 & chromosome partitioning protein \\
\hline $4,722,828,-$ & porB & 22.6 & 2-oxoacid ferredoxin oxidoreductase subunit beta \\
\hline $3,034,066,-$ & MMAR_2491 & 18.2 & zinc ribbon domain-containing protein \\
\hline $5,952,502,+$ & MMAR_4896 & 16.7 & hypothetical protein \\
\hline $2,306,716,+$ & $\operatorname{infB}$ & 11.5 & translation initiation factor IF-2 \\
\hline $1,233,867,+$ & MMAR_1007 & 10.4 & transcriptional regulator \\
\hline $2,424,872,-$ & $\operatorname{sig} B$ & 10.4 & RNA polymerase sigma factor \\
\hline $4,231,315,-$ & MMAR_3428 & 10.1 & hypothetical protein \\
\hline $1,233,815,-$ & mmpS5 & 9.6 & membrane protein \\
\hline $3,478,075,+$ & MMAR_2872 & 8.4 & multidrug-transport integral membrane protein \\
\hline $5,945,575,-$ & purL & 7.9 & phosphoribosylformylglycinamidine synthase subunit \\
\hline $6,373,356,+$ & MMAR_5280 & 7.3 & dihydrodipicolinate reductase \\
\hline \multicolumn{4}{|l|}{ downregulated } \\
\hline $3,623,295,-$ & MMAR_2999 & 0.016 & OsmC family peroxiredoxin \\
\hline $2,115,027,+$ & fadD29 & 0.054 & acyl-CoA synthetase \\
\hline $5,624,834,+$ & $m n t H$ & 0.072 & divalent metal cation transporter \\
\hline $3,801,485,-$ & MMAR_3116 & 0.083 & undecaprenyl-diphosphate phosphatase \\
\hline $2,817,295,+$ & fadD25 & 0.084 & acyl-CoA synthetase \\
\hline $2,816,979,-$ & pks5 & 0.095 & probable polyketide synthase \\
\hline $2,686,224,+$ & MMAR_2235 & 0.095 & PE-PPE domain-containing protein \\
\hline $2,838,256,-$ & MMAR_2352 & 0.097 & GAP family protein \\
\hline $2,966,269,+$ & phet & 0.103 & phenylalanine-tRNA ligase subunit beta \\
\hline $1,541,144,+$ & fadE25 & 0.117 & acyl-CoA dehydrogenase \\
\hline $5,174,763,-$ & $m d h$ & 0.124 & malate dehydrogenase \\
\hline $572,814,-$ & MMAR_5555 & 0.130 & hypothetical protein \\
\hline $3,298,910,+$ & $g c v H$ & 0.132 & glycine cleavage system protein $\mathrm{H}$ \\
\hline $4,257,552,+$ & MMAR_3453 & 0.136 & pyridoxal phosphate-dependent aminotransferase \\
\hline $1,249,089,+$ & MMAR_1023 & 0.137 & mycofactocin biosynthesis peptidyl-dipeptidase \\
\hline
\end{tabular}

${ }^{a}$ pTSS locates in plasmid (NC_010604.1)

and supported our proposal that combining regular RNA-seq with Cappable-seq could provide a means to globally investigate posttranscriptional regulation.

\section{Discussion}

M. marinum has been used as a surrogate for Mtb [27, 39, 40]. Compared with M. smegmatis, M. marinum is more closely genetically related to $\mathrm{Mtb}$, and an $M$. marinum-zebrafish infection model has been established to study latent infection [28, 41]. Recently, genome-scale mapping of TSSs in M. smegmatis during aerobic growth and under hypoxia has been reported [26], but how hypoxic stress affects the strength of TSSs in M. marinum is still unclear. In this study, we applied Cappable-seq to quantifiably identify the changes in TSSs in M. marinum and globally analyze transcriptional regulation in response to hypoxic stress. We totally identified 6808 TSSs. Among them the strength of 1112 TSSs were upregulated and 1265 TSSs were downregulated under hypoxic stress. Moreover, comparing the performance of Cappable-seq and regular RNA-seq, we demonstrated that Cappable-seq is a valuable method for global transcriptional analysis.

Developed from regular RNA-seq, several other modified methods have been performed to study specific characteristics of the transcriptome. dRNA-seq and 

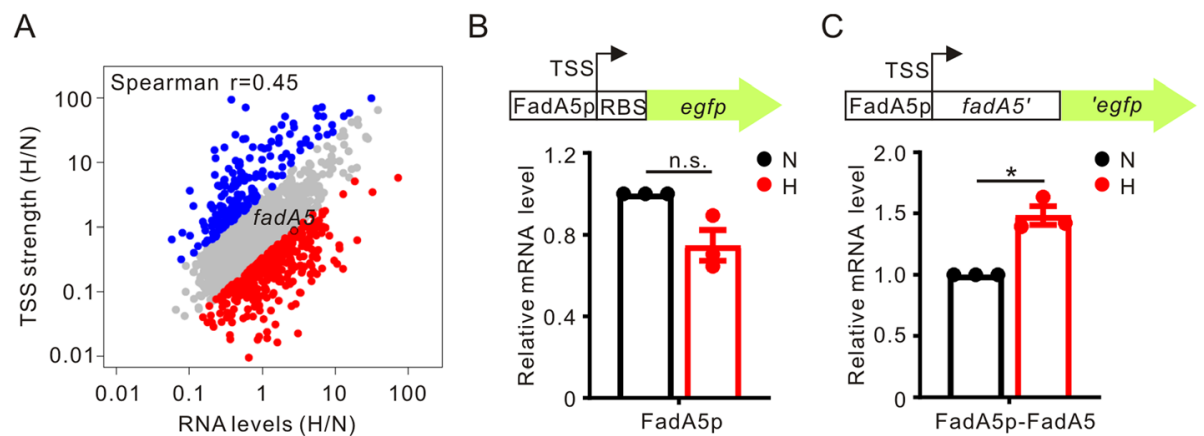

Fig. 5 Comparison of regular RNA-seq and Cappable-seq in global transcriptional analyses. a Correlation between fold-changes of RNA levels and TSS strength under hypoxia $(\mathrm{H})$ compared with normoxia (N) from regular RNA-seq and Cappable-seq data. The graph containing 2387 pTSSs expressed under both normoxia and hypoxia and the expression of downstream genes of these pTSSs were detected by regular RNA-seq. The red and blue dots indicate 393 upregulated and 175 downregulated genes that probably subjected to posttranscriptional regulation. The fad5A gene was chosen for verification using qRT-PCR. b and c Transcriptional (b) and translational (c) fusions of fadA5 to egfp. The primers for qRT-PCR were designed according to the egfp sequence. Relative transcriptional levels under normoxic conditions were normalized to 1. Data are shown as the mean \pm SEM values from biological triplicates performed in duplicate. ${ }^{*}, p<0.05$; n.s., not significant

term-seq have been used to identify the $5^{\prime}$ and 3' boundaries of transcripts, respectively, at genome-scale $[20,42]$. Cappable-seq is a recently developed highthroughput method to determine TSSs, which has been applied in E. coli, Streptococcus pneumoniae and Photorhabdus luminescens [21, 43, 44]. In this study, nearly half of TSSs characterized by Cappable-seq were also identified by dRNA-seq analysis [24], suggesting that these methods are highly reliable and reproducible. In addition, Cappable-seq was reported to have higher sensitivity than dRNA-seq, and thus could reveal novel TSSs that are weakly expressed in E. coli [21]. In $M$. marinum, we also found a higher number of TSSs in Cappable-seq specific TSS that were not identified in dRNA-seq [24].

Recently, the transcriptional regulation of $M$. marinum at different time points of resuscitation from hypoxia-induced dormancy has been characterized [45], which revealed that 8 transcription factors, i.e., MMAR_0923, MMAR_1394, MMAR_1653, MMAR_ 4219, MMAR_4852 (kmtR), MMAR_4874 (cosR), MMAR_5170 (whiB4) and MMAR_5405 (ethR), were significantly downregulated upon resuscitation from dormancy. In our study, 4 of these transcription factors (MMAR_0923, MMAR_4219, kmtR and cosR) were highly upregulated in regular RNA-seq (Table S1) and 5 of these genes (MMAR_0923, MMAR_1394, MMAR_1653, cosR and $k m t R)$ contained TSSs that were upregulated under hypoxic conditions (Table S2), confirming the importance of these transcription factors in coping with hypoxic stress. Importantly, our Cappable-seq data provided the sequences of these regulated promoters, which could facilitate research on target identifications as well as functional analyses of these transcription factors.
Several studies have performed transcriptomic analyses of Mtb under hypoxic conditions [9, 11, 46]. The expression of transcription factors, such as Rv0081, Rv1353c and $\cos R$ ( $R v 0967)$, was reported to be induced in response to hypoxia $[9,11,46]$. In our Cappable-seq data, pTSSs of these homologous genes (MMAR_1653, MMAR_4019 and MMAR_4874) were identified and the strength of these pTSSs was all upregulated under hypoxia (Table S2). These results suggest that Mtb and $M$. marinum exploit similar transcriptional regulation mechanisms to cope with hypoxic stress. In addition, although SigE was shown to be upregulated under hypoxic conditions [19] and 9 transcriptional units directly regulated by SigE after SDS exposure were defined using a DNA microarray [36], the global regulatory targets of SigE in hypoxic responses are still largely unclear. We identified 4 ( $\operatorname{sig} B, h t p X$, fxs $A$ and $\operatorname{clg} R$ ) of 9 pTSSs previously defined as SigE regulatory units, which illustrated the reliability of transcriptional regulation characterization by Cappable-seq. Moreover, we identified 59 SigE-dependent TSSs, which broadened the knowledge related to the regulatory role of SigE in hypoxic responses.

Leaderless transcripts have been identified in different bacterial species. In our study, we found that over $38 \%$ of pTSSs initiate leaderless transcripts in $M$. marinum, higher than the frequencies in Mtb and M. smegmatis (approximately 25\%) [22, 23, 26]. Further studies are needed to elucidate the roles of these leaderless transcripts and the differences of M. marinum with Mtb and M. smegmatis. Moreover, we found that transcripts with 5 ' UTR of 4 nt or longer would not be efficiently translated (Fig. 3c); the role of this phenomenon would be worthy of further study. A recent study tried to elucidate the impact of leaderless transcript structures on 
translation efficiency, transcript stability, and transcription rates in M. smegmatis [47], but the roles of leaderless transcripts remain to be further investigated.

In summary, our study globally mapped the transcriptional regulation of $M$. marinum in response to hypoxia, providing valuable information for future studies of how Mtb transits into dormancy and potential new approach for global transcriptional investigations.

\section{Materials and methods}

\section{Bacterial strains and growth conditions}

The bacterial strains and plasmids used in this study are listed in Table S4 and oligonucleotides are summarized in Table S5. M. marinum cells were grown in Middlebrook 7H9 broth (Difco) supplemented with $0.5 \%(\mathrm{~V} / \mathrm{V})$ glycerol, $0.05 \%$ (W/V) Tween-80 and 10\% (V/V) OADC (Difco) at $32{ }^{\circ} \mathrm{C}, 100 \mathrm{rpm}$ or on Middlebrook $7 \mathrm{H} 10$ agar (Difco) supplemented with $0.5 \%(\mathrm{~V} / \mathrm{V})$ glycerol and $10 \%$ (V/V) OADC (Difco). M. smegmatis used for the eGFP reporter assay was cultured in 7H9 broth supplemented with $0.2 \%(\mathrm{~V} / \mathrm{V})$ glycerol, $0.05 \%(\mathrm{~W} / \mathrm{V})$ Tween-80, $0.2 \%$ $(\mathrm{W} / \mathrm{V})$ glucose and $15 \mathrm{mM} \mathrm{NaCl}$ at $37^{\circ} \mathrm{C}, 200 \mathrm{rpm}$ or on 7H10 agar plates supplemented with $0.5 \%$ glycerol. $E$. coli DH5 $\alpha$ used for cloning was routinely grown at $37^{\circ} \mathrm{C}$ in Luria-Bertani (LB) broth or on LB agar plates. The concentration of kanamycin used was $20 \mu \mathrm{g} / \mathrm{ml}$ for mycobacteria and $50 \mu \mathrm{g} / \mathrm{ml}$ for E. coli $\mathrm{DH} 5 \alpha$.

\section{Hypoxic treatment of $M$. marinum}

The Wayne model [6] was applied for hypoxic treatment. Briefly, $M$. marinum and its derivative strains were aerobically grown to mid-exponential phase $\left(\mathrm{OD}_{600}=0.6\right)$, and then diluted into fresh media containing $1.5 \mu \mathrm{g} / \mathrm{ml}$ methylene blue to a starting $\mathrm{OD}_{600}$ of 0.05 . A volume of $24 \mathrm{ml}$ diluted culture was transferred into a $36 \mathrm{ml} \mathrm{screw-cap}$ tube $(2.5 \mathrm{~cm} \times 8 \mathrm{~cm})$. The solid cap with a latex liner was tightly screwed down and sealed with paraffin wax. After $24 \mathrm{~h}$ of incubation, the methylene blue had become completely decolorized, and the $\mathrm{OD}_{600}$ had reached 0.3. For the normoxic control, $96 \mathrm{ml}$ of the same diluted culture was aerobically incubated in a 250 $\mathrm{ml}$ conical flask. Samples were also collected when the bacterial $\mathrm{OD}_{600}$ reached 0.3.

\section{M. marinum survival under hypoxic stress}

M. marinum cultures prior to and after $24 \mathrm{~h}$ of hypoxic incubation were collected and serially diluted 10-fold. Specifically, $50 \mu \mathrm{l}$ samples were diluted with $450 \mu \mathrm{l}$ $\mathrm{PBST}_{80}$ (PBS supplemented with $0.05 \%$ Tween-80). Then, $100 \mu \mathrm{l}$ aliquots of $10^{-4}$ or $10^{-5}$ dilution were spread onto $7 \mathrm{H} 10$ plates in triplicate. After 7 days of incubation at $32{ }^{\circ} \mathrm{C}$, the number of colonies was counted. These tests were independently performed in triplicate.

\section{RNA extraction and qRT-PCR}

RNA extraction and qRT-PCR were performed as described previously $[15,40]$. Briefly, four copies of $24 \mathrm{ml}$ of M. marinum subjected to hypoxic treatment or $96 \mathrm{ml}$ M. marinum growing under normoxic conditions were harvested by centrifugation at $7500 \mathrm{rpm}$ for $10 \mathrm{~min}$ at $4{ }^{\circ} \mathrm{C}$ and then frozen immediately in liquid nitrogen. After grinding in liquid nitrogen, the samples were transferred to $2 \mathrm{ml}$ tubes containing $1 \mathrm{ml}$ TRIzol (Invitrogen). RNA was extracted according to the manufacturer's protocol. For qRT-PCR experiments, $2 \mu \mathrm{g}$ of RNA was digested with RNase-free DNase I (Promega) and then reverse transcribed to cDNA using M-MLV reverse transcriptase (Promega). iTaq Universal SYBR Green Supermix (Bio-Rad) was used to perform qRTPCR reactions in duplicate. The relative RNA levels of the tested genes were normalized to the levels of sigA. The mean values and standard error of mean (SEM) from biological triplicates are shown. Data comparisons between two groups were performed using Student's ttest.

\section{Construction of regular RNA-seq libraries and sequencing}

Regular RNA-seq was performed as previously described [48]. The rRNA in total RNA extracted from triplicate normoxic and hypoxic cultures was removed by a Ribooff rRNA Depletion Kit (Vazyme), and libraries were constructed using the NEBNext Ultra Directional RNA Library Prep Kit for Illumina (NEB). Sequencing was performed on the Illumina HiSeq X Ten platform using $2 \times 150 \mathrm{bp}$ paired-end sequencing. Fold changes were calculated by comparing the fragments per kilobase per million mapped fragments (FPKM) between two conditions.

\section{Construction of Cappable-seq libraries and sequencing}

RNA samples from triplicate normoxic and hypoxic cultures were subjected to enrichment for primary transcripts as previously described [21], with minor modifications. First, $1.8 \times$ Agencourt AMPure XP beads (Beckman) were used to purify RNA in all procedures. Second, to obtain enough primary transcripts for subsequent library construction, enrichment procedure using hydrophilic streptavidin magnetic beads (NEB) was performed for one time. The NEBNext Small RNA Library Prep Set for Illumina (NEB) was used to generate Cappable-seq libraries. To reduce the concentration of adaptor dimer, the $3^{\prime}$ SR adaptor and $5^{\prime}$ SR adaptor were both used at 4-fold dilutions. After 21 cycles of PCR amplification, the libraries were purified using a QIAquick PCR Purification Kit (QIAGEN) and 1.5× Agencourt AMPure XP beads (Beckman). The concentration and size distribution of the libraries were determined by Qubit 3 (Invitrogen) and Bioanalyzer DNA 
1000 (Agilent) respectively. Sequencing was performed by the Illumina HiSeq $\mathrm{X}$ Ten platform.

\section{Identification and classification of TSSs}

Clean reads of Cappable-seq were mapped to the $M$. marinum strain $\mathrm{M}$ ATCC BAA-535A genome (NC 010612.1 and NC_010604.1). The first nucleotides at the 5 ' ends of the mapped reads were extracted as candidate TSSs. Candidate TSSs located within genes encoding rRNA or tRNA were excluded from further analyses. Based on the number of reads mapped to the subsequent $50 \mathrm{bp}$, the strength of candidate TSSs was calculated as the relative read score (RRS) according to a previous description [21]. Briefly, RRS was calculated as $(\mathrm{Rns} / \mathrm{Rt}) \times 10^{6}$, with Rns being the number of trimmed reads mapped to position $\mathrm{n}$ in the $M$. marinum genome on either strand $(-$ or + ) and Rt being the total number of reads mapped to the $M$. marinum genome. TSSs were classified into five categories according to genome position and TSS strength as previously described [20]. A TSS was classified as a primary TSS when it was located within $\leq 500 \mathrm{bp}$ upstream of the annotated ORF. A secondary TSS was associated with the same ORF but had a lower RRS than the primary TSS. Internal TSSs assigned to TSSs were located inside an ORF on the same strand, and antisense TSSs were also located inside an ORF but on the opposite strand. A TSS was classified as an orphan TSS when it could not be assigned to any of the above four categories.

\section{Promoter motif search}

To analyze the promoter motifs, 12 bp ( -5 to -16 relative to TSS) and $16 \mathrm{bp}$ ( -25 to -40 relative to TSS) sequences of promoters were extracted to search for -10 and - 35 motifs, respectively, using MEME version 5.3.0 (http://meme-suite.org/tools/meme) online [30, 31].

\section{Construction of plasmids}

The eGFP reporter plasmids were constructed by inserting a fragment containing a synthetic promoter and egfp gene into the integrative plasmid pMV306 [38]. Different lengths of 5' UTRs were introduced using the QuikChange II XL site-directed mutagenesis kit (Stratagene). Two aspects are considered for the introduced 5' UTR sequences. First, the introduction of these sequences would not create potential ribosomal binding sites. Second, the introduction of these sequences would not change the secondary structure of egfp mRNA. Fragments of the $f a d A 5$ gene were amplified from the genomic DNA of $M$. marinum strain M ATCC BAA-535A. PCR fragments were inserted into the linearized promoterless vector pMV306-egfp using a ClonExpress II One Step Cloning Kit (Vazyme).

\section{eGFP reporter assay}

The eGFP reporter plasmids were transformed into $M$. smegmatis. Transformants from $7 \mathrm{H} 10$ agar plates were grown to an $\mathrm{OD}_{600}$ of 0.8 in $7 \mathrm{H} 9$ medium, harvested and resuspended in PBST. Cells were transferred to black 96-well plates to test the fluorescence intensities using Bio-TEK Synergy HT (Bio TEK) as described previously [33]. The eGFP expression levels are indicated by relative fluorescence units (RFU, fluorescence intensities per $\left.\mathrm{OD}_{600}\right)$. Two transformants in each group were tested in duplicate. Experiments were independently performed twice.

\section{Comparison of Cappable-seq and regular RNA-seq data}

To test the efficiency of Cappable-seq in characterizing transcriptional regulation, pTSSs expressed under both normoxic and hypoxic conditions were chosen, and then the expression of genes downstream of these pTSSs was determined in regular RNA-seq. For comparison of the fold-changes of RNA levels with pTSS strengths, GraphPad Prism 8.0.2 was used to compute the nonparametric Spearman correlation.

\section{Statistical analyses of regular RNA-seq and Cappable-seq data}

For multiple testing correction, FDR-adjusted $p$-values were obtained using DESeq2 [49] implemented in $\mathrm{R}$ (version 3.2.2). Genes or TSSs were considered to be differentially expressed at fold change $\geq 2$ and adjusted $p<$ 0.05 .

\section{Supplementary Information}

The online version contains supplementary material available at https://doi. org/10.1186/s12864-021-07572-8.

\section{Additional file 1. \\ Additional file 2 . \\ Additional file 3. \\ Additional file 4. \\ Additional file $\mathbf{5}$.}

\section{Acknowledgements}

We thank Dr. Jun Liu from University of Toronto for providing the $M$. marinum ATCC BAA-535 strain, Dr. Jiaoyu Deng from WIV for providing $M$. smegmatis mc2155 strain, Dr. K Heran Darwin from New York University for providing pMV306 plasmid, and Lei Zhang from the Core Facility and Technical Support of WIV for help in preparing libraries for RNA-seq analyses.

\section{Authors' contributions}

S.H. and W.Z. performed the research and analyzed the data, S.H., Y.H. and S.C. designed the research and wrote the manuscript, W.T. and Y.Z. provided reagents. All authors reviewed the manuscript. The author(s) read and approved the final manuscript.

\section{Funding}

This work was supported by grants from the National Science Foundation of China (\#81861148019 to S.C and \#31870133 to Y.H.) and the Youth Innovation Promotion Association CAS (Y201750, Y.H.). 


\section{Availability of data and materials}

The RAW data for Cappable-seq assays are available in SRA: PRJNA684793. The RAW data for regular RNA-seq assays are available in SRA: PRJNA684796.

\section{Declarations}

Ethics approval and consent to participate

Not applicable.

\section{Consent for publication}

Not applicable.

\section{Competing interests}

The authors declare that there are no conflicts of interest.

\section{Author details}

${ }^{1}$ CAS Key Laboratory of Special Pathogens and Biosafety, Wuhan Institute of Virology, Center for Biosafety Mega-Science, Chinese Academy of Sciences, Wuhan 430071, China. ${ }^{2}$ University of Chinese Academy of Sciences, Beijing 100049, China.

\section{Received: 23 December 2020 Accepted: 26 March 2021}

Published online: 06 April 2021

\section{References}

1. Koul A, Arnoult E, Lounis N, Guillemont J, Andries K. The challenge of new drug discovery for tuberculosis. Nature. 2011;469(7331):483-90. https://doi. org/10.1038/nature09657.

2. Gengenbacher M, Kaufmann SH. Mycobacterium tuberculosis: success through dormancy. FEMS Microbiol Rev. 2012;36(3):514-32. https://doi.org/1 0.1111/j.1574-6976.2012.00331.x.

3. Wayne LG, Sohaskey CD. Nonreplicating persistence of mycobacterium tuberculosis. Annu Rev Microbiol. 2001;55(1):139-63. https://doi.org/10.114 6/annurev.micro.55.1.139.

4. Schnappinger D, Ehrt S, Voskuil MI, Liu Y, Mangan JA, Monahan IM, et al. Transcriptional adaptation of Mycobacterium tuberculosis within macrophages: insights into the Phagosomal environment. J Exp Med. 2003; 198(5):693-704. https://doi.org/10.1084/jem.20030846.

5. Rustad TR, Sherrid AM, Minch KJ, Sherman DR. Hypoxia: a window into Mycobacterium tuberculosis latency. Cell Microbiol. 2009;11(8):1151-9. https://doi.org/10.1111/j.1462-5822.2009.01325.x.

6. Wayne LG, Hayes LG. An in vitro model for sequential study of shiftdown of Mycobacterium tuberculosis through two stages of nonreplicating persistence. Infect Immun. 1996;64(6):2062-9. https://doi.org/10.1128/IAl.64. 6.2062-2069.1996.

7. Yuan Y, Crane DD, Simpson RM, Zhu YQ, Hickey MJ, Sherman DR, et al. The $16-k D a$ alpha-crystallin (Acr) protein of Mycobacterium tuberculosis is required for growth in macrophages. Proc Natl Acad Sci U S A. 1998;95(16): 9578-83. https://doi.org/10.1073/pnas.95.16.9578.

8. Sherman DR, Voskuil M, Schnappinger D, Liao R, Harrell MI, Schoolnik GK. Regulation of the Mycobacterium tuberculosis hypoxic response gene encoding alpha -crystallin. Proc Natl Acad Sci U S A. 2001;98(13):7534-9. https://doi.org/10.1073/pnas.121172498.

9. Rustad TR, Harrell MI, Liao R, Sherman DR. The enduring hypoxic response of Mycobacterium tuberculosis. PLoS One. 2008;3(1):e1502. https://doi.org/1 0.1371/journal.pone.0001502.

10. Park HD, Guinn KM, Harrell MI, Liao R, Voskuil Ml, Tompa M, et al. Rv3133c/ dosR is a transcription factor that mediates the hypoxic response of Mycobacterium tuberculosis. Mol Microbiol. 2003;48(3):833-43. https://doi. org/10.1046/j.1365-2958.2003.03474.x.

11. Galagan JE, Minch K, Peterson M, Lyubetskaya A, Azizi E, Sweet L, et al. The Mycobacterium tuberculosis regulatory network and hypoxia. Nature. 2013; 499(7457):178-83. https://doi.org/10.1038/nature12337.

12. Davis MC, Kesthely CA, Franklin EA, MacLellan SR. The essential activities of the bacterial sigma factor. Can J Microbiol. 2017;63(2):89-99. https://doi. org/10.1139/cjm-2016-0576.

13. Ruff EF, Record MT Jr, Artsimovitch I. Initial events in bacterial transcription initiation. Biomolecules. 2015;5(2):1035-62. https://doi.org/10.3390/biom5021 035.

14. Gebhard S, Hümpel A, McLellan AD, Cook GM. The alternative sigma factor SigF of Mycobacterium smegmatis is required for survival of heat shock, acidic pH and oxidative stress. Microbiol (Reading, England). 2008;154(Pt 9): 2786-95.

15. Hu Y, Wang Z, Feng $L$, Chen Z, Mao C, Zhu Y, et al. $\sigma(E)$-dependent activation of RbpA controls transcription of the furA-katG operon in response to oxidative stress in mycobacteria. Mol Microbiol. 2016;102(1): 107-20. https://doi.org/10.1111/mmi.13449.

16. Manganelli R. Sigma factors: key molecules in Mycobacterium tuberculosis physiology and virulence. Microbiol Spectrum. 2014;2(1):MGM2 -0007-2013.

17. Rodrigue S, Provvedi R, Jacques PE, Gaudreau L, Manganelli R. The sigma factors of Mycobacterium tuberculosis. FEMS Microbiol Rev. 2006;30(6):92641. https://doi.org/10.1111/j.1574-6976.2006.00040.x.

18. Wu QL, Kong D, Lam K, Husson RN. A mycobacterial extracytoplasmic function sigma factor involved in survival following stress. J Bacteriol. 1997; 179(9):2922-9. https://doi.org/10.1128/JB.179.9.2922-2929.1997.

19. Iona E, Pardini M, Mustazzolu A, Piccaro G, Nisini R, Fattorini L, et al. Mycobacterium tuberculosis gene expression at different stages of hypoxiainduced dormancy and upon resuscitation. J Microbiol (Seoul, Korea). 2016; 54(8):565-72.

20. Sharma CM, Hoffmann S, Darfeuille F, Reignier J, Findeiss S, Sittka A, et al. The primary transcriptome of the major human pathogen helicobacter pylori. Nature. 2010;464(7286):250-5. https://doi.org/10.1038/nature08756.

21. Ettwiller L, Buswell J, Yigit E, Schildkraut I. A novel enrichment strategy reveals unprecedented number of novel transcription start sites at single base resolution in a model prokaryote and the gut microbiome. BMC Genomics. 2016;17(1):199. https://doi.org/10.1186/s12864-016-2539-z.

22. Cortes T, Schubert OT, Rose G, Arnvig KB, Comas I, Aebersold R, et al. Genome-wide mapping of transcriptional start sites defines an extensive leaderless transcriptome in Mycobacterium tuberculosis. Cell Rep. 2013;5(4): 1121-31. https://doi.org/10.1016/j.celrep.2013.10.031.

23. Shell SS, Wang J, Lapierre P, Mir M, Chase MR, Pyle MM, et al. Leaderless transcripts and small proteins are common features of the mycobacterial translational landscape. PLoS Genet. 2015;11(11):e1005641. https://doi.org/1 0.1371/journal.pgen.1005641

24. Dinan AM, Tong P, Lohan AJ, Conlon KM, Miranda-CasoLuengo AA, Malone KM, et al. Relaxed selection drives a noisy noncoding transcriptome in members of the Mycobacterium tuberculosis complex. mBio. 2014:5(4): e01169-14. https://doi.org/10.1128/mBio.01169-14.

25. Li X, Mei H, Chen F, Tang Q, Yu Z, Cao X, et al. Transcriptome landscape of Mycobacterium smegmatis. Front Microbiol. 2017;8:2505. https://doi.org/1 0.3389/fmicb.2017.02505

26. Martini MC, Zhou Y, Sun H, Shell SS. Defining the transcriptional and posttranscriptional landscapes of Mycobacterium smegmatis in aerobic growth and hypoxia. Front Microbiol. 2019;10:591. https://doi.org/10.3389/fmicb.201 9.00591.

27. Davis JM, Ramakrishnan $L$. The role of the granuloma in expansion and dissemination of early tuberculous infection. Cell. 2009;136(1):37-49. https:// doi.org/10.1016/j.cell.2008.11.014.

28. Tobin DM, Ramakrishnan L. Comparative pathogenesis of Mycobacterium marinum and Mycobacterium tuberculosis. Cell Microbiol. 2008;10(5):102739. https://doi.org/10.1111/j.1462-5822.2008.01133.x.

29. Banerjee A, Dubnau E, Quemard A, Balasubramanian V, Um KS, Wilson T, et al. inhA, a gene encoding a target for isoniazid and ethionamide in Mycobacterium tuberculosis. Science (New York, NY). 1994;263(5144):227-30.

30. Bailey TL, Elkan C. Fitting a mixture model by expectation maximization to discover motifs in biopolymers. Proc Int Conf Intell Syst Mol Biol. 1994:2:28-36.

31. Bailey TL, Williams N, Misleh C, Li WW. MEME: discovering and analyzing DNA and protein sequence motifs. Nucleic Acids Research. 2006;34(Web Server issue):W369-73.

32. Agarwal N, Tyagi AK. Role of $5^{\prime}-\mathrm{TGN}-3^{\prime}$ motif in the interaction of mycobacterial RNA polymerase with a promoter of 'extended -10 ' class. FEMS Microbiol Lett. 2003;225(1):75-83. https://doi.org/10.1016/S0378-1 097(03)00483-X

33. Zhu Y, Mao C, Ge X, Wang Z, Lu P, Zhang Y, Chen S, Hu Y. Characterization of a minimal type of promoter containing the -10 element and a guanine at the -14 or -13 position in mycobacteria. J Bacteriol 2017;199(21):e0038517. https://doi.org/10.1128/JB.00385-17.

34. Newton-Foot M, Gey van Pittius NC. The complex architecture of mycobacterial promoters. Tuberculosis (Edinburgh, Scotland). 2013;93(1): 60-74. 
35. Helmann JD. Where to begin? Sigma factors and the selectivity of transcription initiation in bacteria. Mol Microbiol. 2019;112(2):335-47. https:// doi.org/10.1111/mmi.14309.

36. Manganelli R, Voskuil MI, Schoolnik GK, Smith I. The Mycobacterium tuberculosis ECF sigma factor sigmaE: role in global gene expression and survival in macrophages. Mol Microbiol. 2001;41(2):423-37. https://doi.org/1 0.1046/j.1365-2958.2001.02525.x.

37. Song T, Song SE, Raman S, Anaya M, Husson RN. Critical role of a single position in the -35 element for promoter recognition by Mycobacterium tuberculosis SigE and SigH. J Bacteriol. 2008;190(6):2227-30. https://doi. org/10.1128/JB.01642-07.

38. Stover CK, de la Cruz VF, Fuerst TR, Burlein JE, Benson LA, Bennett LT, et al. New use of BCG for recombinant vaccines. Nature. 1991;351 (6326):456-60. https://doi.org/10.1038/351456a0.

39. Bosserman RE, Nguyen $T$, Sanchez $K G$, Chirakos $A E$, Ferrell MJ, Thompson CR, et al. WhiB6 regulation of ESX-1 gene expression is controlled by a negative feedback loop in Mycobacterium marinum. Proc Natl Acad Sci U S A. 2017;114(50):E10772-81. https://doi.org/10.1 073/pnas.1710167114.

40. Chen Z, Hu Y, Cumming BM, Lu P, Feng L, Deng J, et al. Mycobacterial WhiB6 differentially regulates ESX-1 and the dos Regulon to modulate granuloma formation and virulence in Zebrafish. Cell Rep. 2016;16(9):251224. https://doi.org/10.1016/j.celrep.2016.07.080.

41. Stinear TP, Seemann T, Harrison PF, Jenkin GA, Davies JK, Johnson PD, et al. Insights from the complete genome sequence of Mycobacterium marinum on the evolution of Mycobacterium tuberculosis. Genome Res. 2008;18(5): 729-41. https://doi.org/10.1101/gr.075069.107.

42. Dar D, Shamir M, Mellin JR, Koutero M, Stern-Ginossar N, Cossart P, et al. Term-seq reveals abundant ribo-regulation of antibiotics resistance in bacteria. Science (New York, NY). 2016;352(6282):aad9822.

43. Slager J, Aprianto R, Veening JW. Deep genome annotation of the opportunistic human pathogen Streptococcus pneumoniae D39. Nucleic Acids Res. 2018;46(19):9971-89. https://doi.org/10.1093/nar/gky725.

44. Tobias NJ, Linck A, Bode HB. Natural product diversification mediated by alternative transcriptional starting. Angewandte Chemie (International ed in English). 2018:57(20):5699-702. https://doi.org/10.1002/anie.201713199.

45. Jiang J, Lin C, Zhang J, Wang Y, Shen L, Yang K, et al. Transcriptome changes of Mycobacterium marinum in the process of resuscitation from hypoxia-induced dormancy. Front Genet. 2019;10:1359.

46. Peterson EJR, Abidi AA, Arrieta-Ortiz ML, Aguilar B, Yurkovich JT, Kaur A, et al. Intricate genetic programs controlling dormancy in Mycobacterium tuberculosis. Cell Rep. 2020;31(4):107577. https://doi. org/10.1016/j.celrep.2020.107577.

47. Nguyen TG, Vargas-Blanco DA, Roberts LA, Shell SS. The impact of leadered and leaderless gene structures on translation efficiency, transcript stability, and predicted transcription rates in mycobacterium smegmatis. J Bacteriol 2020;202(9):e00746-19. https://doi.org/10.1128/JB.00746-19.

48. Shi W, Zhou W, Zhang B, Huang S, Jiang Y, Schammel A, et al. Structura basis of bacterial $\sigma(28)$-mediated transcription reveals roles of the RNA polymerase zinc-binding domain. EMBO J. 2020;39(14):e104389. https://doi. org/10.15252/embj.2020104389

49. Love Ml, Huber W, Anders S. Moderated estimation of fold change and dispersion for RNA-seq data with DESeq2. Genome Biol. 2014;15(12):550. https://doi.org/10.1186/s13059-014-0550-8.

\section{Publisher's Note}

Springer Nature remains neutral with regard to jurisdictional claims in published maps and institutional affiliations.

Ready to submit your research? Choose BMC and benefit from:

- fast, convenient online submission

- thorough peer review by experienced researchers in your field

- rapid publication on acceptance

- support for research data, including large and complex data types

- gold Open Access which fosters wider collaboration and increased citations

- maximum visibility for your research: over $100 \mathrm{M}$ website views per year

At BMC, research is always in progress.

Learn more biomedcentral.com/submissions 